\title{
Damage law identification of a quasi brittle ceramic from a bending test using digital image correlation
}

\author{
P. Leplay ${ }^{1,2,3, a}$, J. Réthoré ${ }^{1}$, S.Meille $^{2}$, and M.-C. Baietto ${ }^{1}$ \\ 1 Université de Lyon, CNRS INSA-Lyon, LaMCoS UMR 5259, 69621 Villeurbanne, France \\ 2 Université de Lyon, CNRS INSA-Lyon, MATEIS UMR 5510, 69621 Villeurbanne, France \\ 3 Saint-Gobain, CREE, 550 av. Alfred Jauffret - BP 224, 84306 Cavaillon, France
}

\begin{abstract}
The quasi brittle ceramics show a non linear mechanical behaviour resulting most of the time in a dissymetry between their tensile and compressive stress-strain laws. The characterization of their fracture strengths might be biased if elastic linear formulae are used to analyze classical tests like bending tests. Based on Digital Image Correlation (DIC), a methodology is proposed to characterize materials with dissymmetric behaviours. Applying specific DIC decomposition functions for bending, compressive and tensile tests, a stress-strain model and its damage law are identified for aluminium titanate, a damageable micro cracked ceramic. This identification method using DIC can obviously be applied to other quasi brittle materials.
\end{abstract}

\section{Introduction}

Some of ceramics are quasi brittle and show a non linear mechanical behaviour. Their quasi brittleness results from the evolution of multiple internal mechanisms. We can mention the most common mechanism: the micro crack propagation for natural rocks [1] or ceramics made of crystals with anisotropic thermal expansion coefficients [2,3]. Other internal mechanisms may occur for bones, plasters, zirconia, refractories and concrete. A dissymmetric evolution of these mechanisms might appear between tension and compression, leading to differences of both constitutive laws and fracture strengths for tensile and compressive loadings.

Experimentally, bending tests are often preferred in the literature and in the industry to measure fracture strengths. But the load-displacement curves of damageable ceramics are usually analyzed in the symmetric elastic linear context. Therefore the determination of the fracture strengths might be biased for such quasi brittle ceramics.

Obtaining reliable uniaxial results through bending tests is not a new idea but phenomenological approaches with inverse methods are usually preferred [4]. Instead of using numerical inverse methods, it is here preferred to directly determine a mechanical model suitable for tension and compression. This last requirement is particularly important for finite element simulations.

To measure strains during bending tests, the traditional way is to use gauges or extensometers. Digital Image Correlation (DIC) is here preferred since it offers the capacity to access the full displacement field on the whole sample surface [5]. This technique based on the optical flow conservation enables the identification of elastic properties [6]. The decomposition adopted for the searched displacement field is a key step for an accurate displacement measurement [7]. The aim of the methodology proposed in this work is to develop new specific decomposition functions for DIC adapted for bending and uniaxial tests (Section 2) in order to identify the

\footnotetext{
${ }^{\mathrm{a}}$ e-mail: paul.leplay@insa-lyon.fr
}

This is an Open Access article distributed under the terms of the Creative Commons Attribution-Noncommercial License 3.0, which permits unrestricted use, distribution, and reproduction in any noncommercial medium, provided the original work is properly cited. 
compressive and tensile constitutive laws and fracture stengths for damageable ceramics (Sections 3 and 4). This methodology is illustrated through an application on aluminium titanate, a micro cracked ceramic whose mechanical and fracture aspects have rather little been investigated in literature.

\section{Digital Image Correlation Developments}

\subsection{General Principle: Optical Flow Conservation}

DIC is a full field measurement method which enables one to capture both local events and global gradients for the displacement of a structure. It is based on the grey level conservation principle between two images of a same structure. Let us call $f$ the reference image, $g$ the deformed image, $u$ the displacement field. This technique consists in comparing two successive images to find the displacement field thanks to the optical flow conservation for each pixel of coordinate $x$ :

$$
f(x)=g(x+u(x))
$$

To determine $v$, the best approximation possible for $u$, one can solve this ill-posed inverse problem on chosen regions $\Omega$ by minimizing the global error thanks to the functional $\phi$ :

$$
\phi^{2}(x)=\iint_{\Omega}[f(x)-g(x+v(x))]^{2} d x
$$

The displacement field is decomposed as a linear combination of basis functions $N_{i}$ :

$$
v(x)=\sum_{i} \alpha_{i} N_{i}(x)
$$

The displacement field decomposition in equation (3) is one of the key tools of DIC. It enables one to introduce mechanical hypotheses into a numerical problem leading to a regularization of the displacement solution $v$. Following this concept, specific basis function sets have already been proposed in the literature: Besnard et al. for arbitrary mechanical problem with bi-linear Q4 elements [8], Hild et al. for cantilever beam kinematics with a linear curvature element [7], Roux et al. for stress intensity factor measurements [9]. Two new sets of specific functions are now proposed for two particular cases: four-point bending tests with a constant curvature element (Section 2.2) and uniaxial tests with a homogeneous strain element (Section 2.3).

\subsection{Particular Case: Four-Point Bending Kinematics}

The four-point bending test is often used in the scientific literature and the industry to characterize the material strengths, as it requires simple sample geometry and equipment. Assuming the Euler-Bernoulli hypotheses for the four-point bending test, the whole kinematics of the central part can be described with the curvature value and the boundary conditions.

The bi-linear Q4-DIC approach developed by Besnard et al. [8] could also be used for the four-point bending test since it is suitable for all mechanical problems with a continuous displacement field. But two drawbacks have to be underlined. First, the noise sensitivity of this general technique may be too important, especially when the displacements are very small like for ceramics. Second, it is not optimal to average a posteriori the displacement over the beam, since there is no direct access to the curvature due to the vanishing of the second order derivatives of the bi-linear Q4 element.

Therefore developing a specific DIC technique which enables the direct measurement of the 

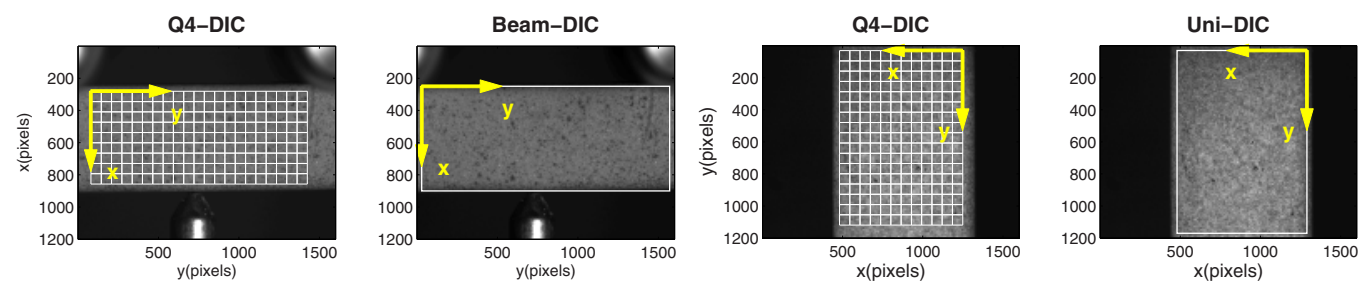

Figure 1. Reference images and zones of interest for four-point bending (left) and compressive (right) tests - Pixel physical size $=10.9 \mu \mathrm{m}$

constant curvature of a beam is a more efficient approach. This goal can be reached thanks to the a priori choice of basis functions for an element with constant second order spatial derivatives.

For a four-point bending test, the displacement field is expressed according to equation (3) as a sum of five degrees of freedom within the region of interest $\Omega$. Assuming a constant beam curvature, three degrees of freedom $\left(\gamma_{0}, \theta_{0}\right.$ and $\left.v_{x 0}\right)$ are required for a two-degree polynomial describing the beam deflection. Two degrees of freedom (dof: $v_{y 0}$ and $\epsilon_{y y}$ ) are introduced to accommodate test imperfections like a neutral axis shift.

$$
\left[\begin{array}{l}
v_{x} \\
v_{y}
\end{array}\right]=\left[\begin{array}{ccccc}
0.5 y^{2} & y & 1 & 0 & 0 \\
y & 1 & 0 & 1 & y
\end{array}\right] \cdot\left[\begin{array}{c}
\gamma_{0} \\
\theta_{0} \\
v_{x 0} \\
v_{y 0} \\
\epsilon_{y y}
\end{array}\right]
$$

Thanks to this specific decomposition, the axial strain becomes directly available with a high accuracy because it is a linear function of the vertical coordinates $x$. The approach developed in this section will henceforth be called 'beam-DIC'.

\subsection{Particular Case: Uniaxial Tensile and Compressive Kinematics}

Even if uniaxial tests are more complicated to implement and run than bending tests, they provide direct information about the material behaviour. To measure displacement and strain fields during such uniaxial tests, new specific functions adapted to the test kinematics are proposed. During tensile or compressive tests, the displacement kinematics can be decomposed with six degrees of freedom. Assuming homogeneous strain on the sample surface, three degrees $\left(\epsilon_{x x}, \epsilon_{y y}\right.$ and $\left.\epsilon_{x y}\right)$ are necessary for in-plane strains. Three degrees of freedom are added for the description of the vertical and horizontal translations $\left(v_{x 0}\right.$ and $\left.v_{y 0}\right)$ and for in-plane rotation $\left(\theta_{x y}\right)$.

$$
\left[\begin{array}{l}
v_{x} \\
v_{y}
\end{array}\right]=\left[\begin{array}{cccccc}
x & 0 & 0.5 y & y & 1 & 0 \\
0 & y & 0.5 x & -x & 0 & 1
\end{array}\right] \cdot\left[\begin{array}{l}
\epsilon_{x x} \\
\epsilon_{y y} \\
\epsilon_{x y} \\
\theta_{x y} \\
v_{0 x} \\
v_{0 y}
\end{array}\right]
$$

This specific decomposition enables a direct access to the homogeneous strain value for compressive or tensile tests without cumbersome averaging post treatment. The approach developed in this section will henceforth be called 'uni-DIC'. 


\section{Samples, Mechanical Tests and Optical EquipmentExperimental Procedure}

Aluminium titanate is known to have a damageable mechanical behaviour at room temperature due to the micro crack propagation [10]. The tested material is mainly made of aluminium titanate with a secondary silicate phase. It is a highly porous ceramic with a porosity between 40 and $50 \%$. The samples were extruded then sintered for a final rectangular section of $\mathrm{B} \times \mathrm{W}=5.1 \times 7.3 \mathrm{~mm}^{2}$. Five samples were used for each test:

- Four-point bending tests were performed on a hydraulic machine with a $5000 \mathrm{~N}$ load cell. The lower span was $D_{1}=60 \mathrm{~mm}$ and the upper span $D_{2}=20 \mathrm{~mm}$. A linear variable differential transducer (LVDT) was used to control the test at a rate of $0.05 \mathrm{~mm} / \mathrm{min}$.

- Compressive tests were performed on the same hydraulic machine, load cell and crosshead speed. The sample length was about $25 \mathrm{~mm}$.

- Tensile tests were performed on an electromechanical machine with a $500 \mathrm{~N}$ load cell and a $0.02 \mathrm{~mm} / \mathrm{min}$ deflection speed. The samples were about $20 \mathrm{~mm}$ long and were stuck to the fixtures thanks to a slow-setting glue likely to penetrate into the material pores. - A CCD camera has been used to visualize the sample surface during each mechanical test. The maximum image resolution of this camera is $1200 \times 1600$ pixels with an 8-bit digitization for grey levels. The acquisition frequency was one image every five seconds. A painted random pattern was spread on the sample surface to enhance the image contrast. Each pixel has a $10.9 \mu \mathrm{m}$ physical size with a $200 \mathrm{~mm}$ telecentric lens.

\section{Results For Aluminium Titanate}

\subsection{Four-Point Bending Test}

Figure 2 shows a non linear load-deflection curve. The measured maximum load $\mathrm{P}$ is $22.7 \pm 0.4$ $\mathrm{N}$ for a vertical deflection $d$ of $208 \pm 11 \mu \mathrm{m}$ at the middle of the beam. Since ceramics are known to break at low strains, one can be tempted to use elastic linear formulae (6) to analyze this four-point bending test:

$$
\epsilon_{\text {elastic }}=\frac{12 d W}{2 D_{1}^{2}+2 D_{1} D_{2}-D_{2}^{2}} \quad \sigma_{\text {elastic }}=\frac{3 P\left(D_{1}-D_{2}\right)}{2 B W^{2}}
$$

These elastic formulae lead to fracture strengths of 5.0 $\pm 0.1 \mathrm{MPa}$ and failure strains of $0.19 \pm 0.1$ $\%$. But these elastic formulae implicitly assume a symmetry between tension and compression
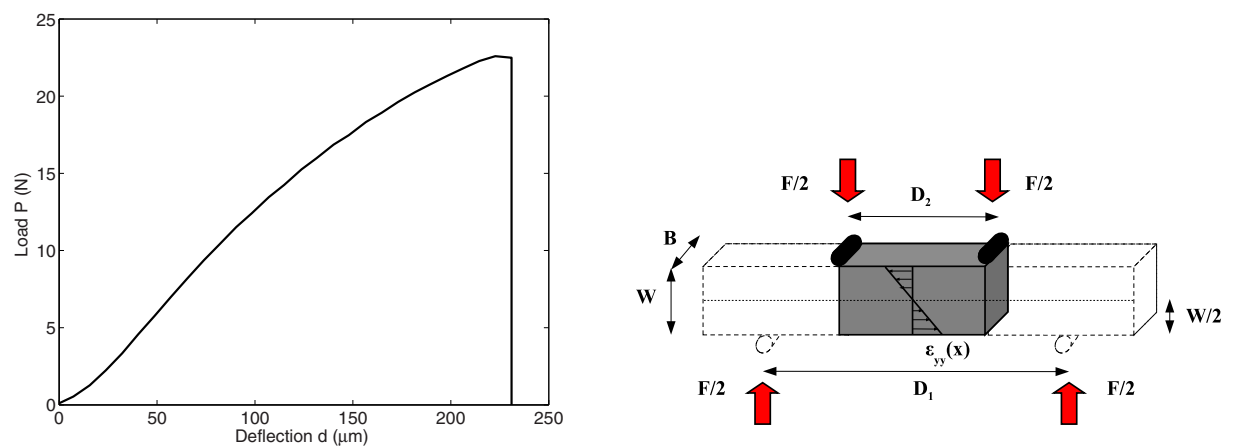

Figure 2. Experimental load-deflection curve for the four-point bending test on aluminium titanate at room temperature (left) - Neutral axis position according to the elastic linear hypotheses (right) 

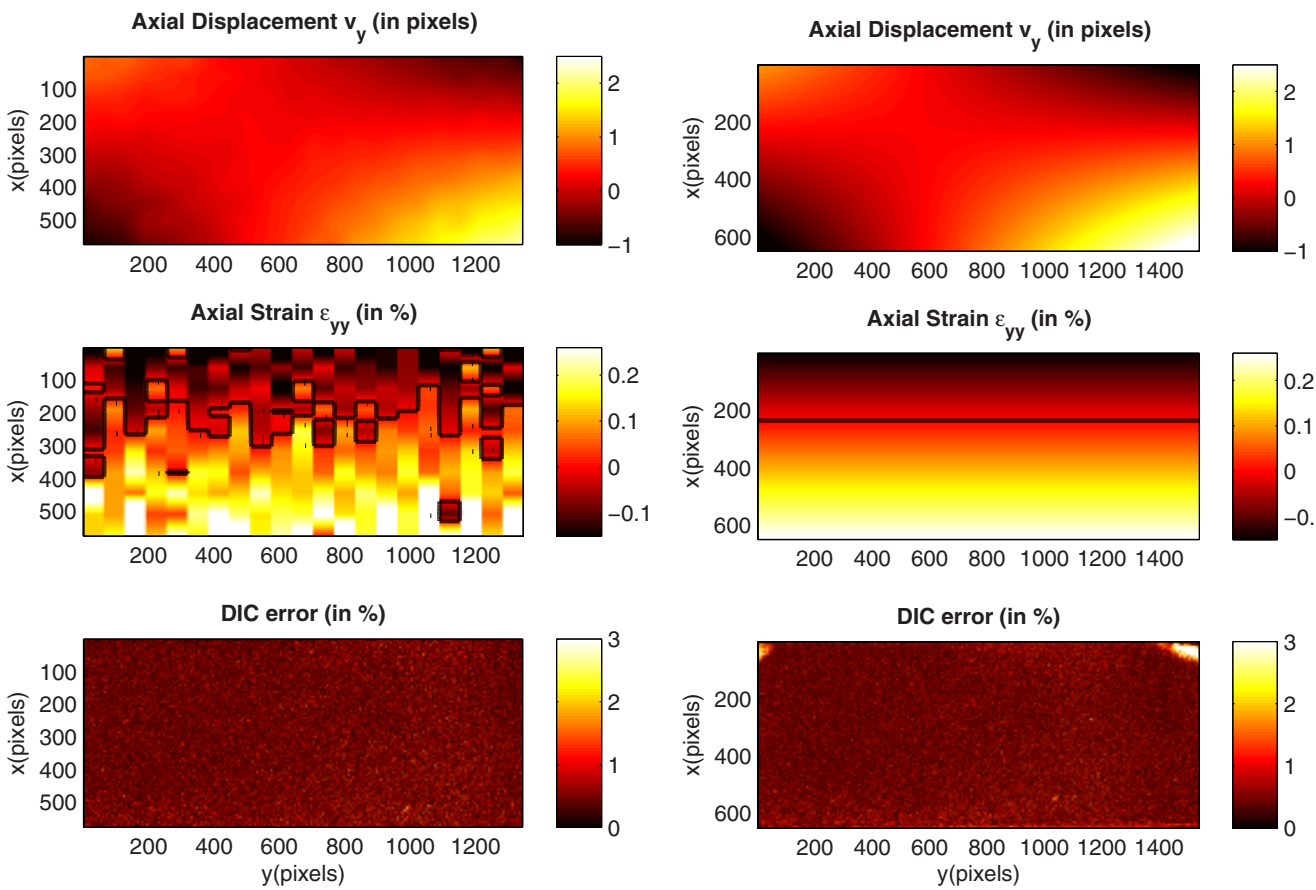

Figure 3. Kinematics measured using the Q4-DIC (left) and beam-DIC (right) approaches just before failure for the bending test - The black line is the iso-0 of $\epsilon_{y y}$

- i.e. a neutral axis located at the mid-height of the beam section (Figure 2). This elastic approach is widely adopted in the literature and in the industry to characterize aluminium titanate at room temperature or at high temperature [10]. It is not possible to quantify the strength of such damageable ceramics from only the deflection and load measurements. Therefore one needs to have access the full strain field during a four-point bending test. The Q4-DIC and beam-DIC approaches are now used to achieve this full field measurement for one of the five samples :

Using the Q4-DIC approach (198 elements / size length $=64$ pixels), the measured displacement field amplitudes $v_{y}$ are so small that the corresponding strain values $\epsilon_{y y}$ are not reliable (Figure 3). Using the beam-DIC approach with one element, the measured displacement field $v_{y}$ and the corresponding axial strain field $\epsilon_{y y}$ are more regular and accurate.

The axial strain field measured during the bending test reveals a dissymmetric behaviour. At the failure load, the beam-DIC approach yields to maximum strains of $+0.26 \%$ for the tensile part and of $-0.15 \%$ for the compressive one. This neutral axis shift during the test indicates a difference between compressive and tensile behaviours.

During the bending test, the correlation error is homogeneous and the mean error remains between 0.5 and $0.6 \%$. This low error value confirms that the kinematics hypotheses of the beam-DIC approach are consistent.

\subsection{Uniaxial Compressive and Tensile Tests}

For the compressive test, the measured maximum stress is 27.5 $\pm 1.1 \mathrm{MPa}$. Even if the Q4-DIC approach (204 elements / size length $=64$ pixels) allows one to identify the axial and shear displacement fields on the sample surface, it is impossible to obtain a reliable value for the 

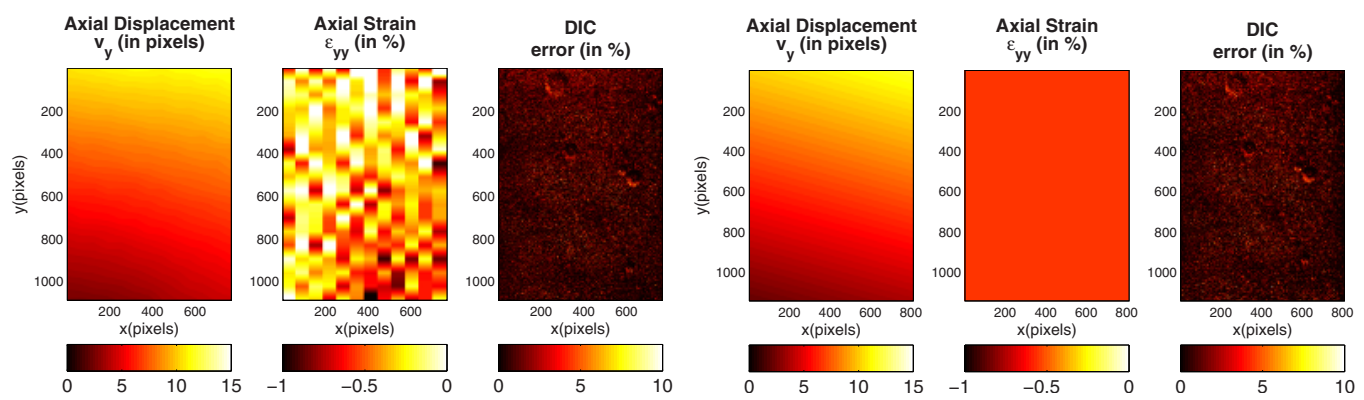

Figure 4. Kinematics measured using the Q4-DIC (left) and uni-DIC (right) approaches for the maximum load in the elastic domain for the compressive test

axial strain (Figure 4). On the contrary, the uni-DIC approach leads directly to a homogeneous strain value: the axial strain at failure is $-0.74 \pm 0.03 \%$.

The stress-strain curve (Figure 5) shows that the mechanical behaviour is elastic linear until a strain of $-0.50 \pm 0.05 \%$ is reached. The secant modulus remains almost equal to $4.6 \mathrm{GPa}$ until this strain limit.

Strains have been quantified according to the beam-DIC approach during the four-point bending test in section 4.1: the value of $-0.15 \%$ was reached in the compressive part of the beam just before the catastrophic failure. It means that the mechanical behaviour for the compressive part of the bent beam can be considered as elastic linear with a Young modulus of $4.6 \mathrm{GPa}$. - For the tensile Test, the measured maximum stress is equal to $3.0 \pm 0.3 \mathrm{MPa}$ for an axial strain of $+0.11 \pm 0.05 \%$ using the uni-DIC approach. The stress-strain curve is not linear and the secant modulus descreases progressively from 4.7 to $2.7 \mathrm{GPa}$. The initial modulus is almost equal to the one measured in compression. This damageable behaviour is linked to the progressive propagation of the grain boundary micro cracks of the aluminium titanate.

The maximum strain is $+0.11 \%$ in the tensile test whereas values of $+0.26 \%$ have been measured during the four-point bending test. Smaller strains in tension than in bending tests have already been observed in literature [4,11]. Beyond the Weibull modulus, it may come from a stress concentration due to inaccurate boundary conditions. An other reason may be a structural effect: the transition from stability to instability depends on the whole stress field of the considered structure [11].
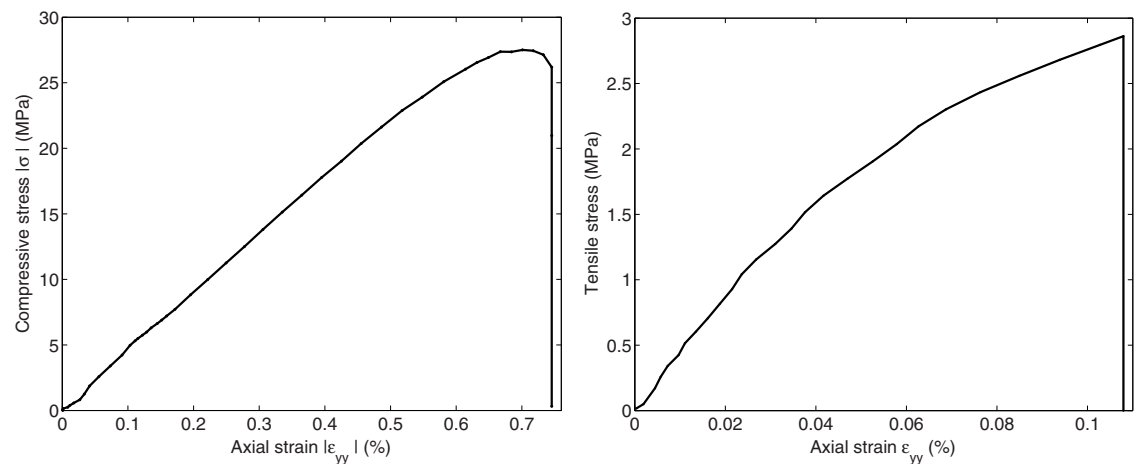

Figure 5. Stress-Strain results obtained using the uni-DIC approach for the compressive (left) and tensile (right) tests on aluminium titanate 


\subsection{Prediction of the Bending Curve through a Mechanical Model}

The aim of this last section is to identify a stress-strain model which can be used henceforth, for instance, in a finite element simulation. The model validation is achieved by predicting the experimental load during a four-point bending test at room temperature.

It has been shown that the mechanical behaviour is elastic for strains from 0.0 to $-0.15 \%$. So an elastic law is well suited to describe the compressive behaviour during a bending test:

$$
\sigma_{\text {compression }}(\epsilon)=E_{0} \cdot \epsilon
$$

It has been shown that the mechanical behaviour is damageable in tension. The fracture strain measured using the uni-DIC approach was $+0.11 \%$ during the tensile test, whereas strains of $+0.26 \%$ obtained using the beam-DIC approach were reached during the bending test. To overcome this lack of experimental data between +0.11 and $+0.26 \%$, a damage law is proposed to describe the tensile behaviour. The micro crack propagation law is represented by an exponential function $D(\epsilon)$ with two new parameters $a$ and $b$. Such exponential damage laws have already been observed on other quasi brittle ceramics [12].

$$
\begin{gathered}
D_{\text {tension }}(\epsilon)=a \cdot(1-\exp (-\epsilon / b)) \\
\sigma_{\text {tension }}(\epsilon)=E_{0} \cdot(1-D(\epsilon)) \cdot \epsilon
\end{gathered}
$$

To identify the numerical values of the three parameters $\left(E_{0}, a, b\right)$, the load applied during the four-point bending test is quantified according to the previous constitutive laws. For every pixel of coordinate $x$, the strain is measured using the beam-DIC approach and the corresponding stress is calculated using equations $(7,8,9)$. Then the load $\mathrm{P}$ is calculated by integrating the moment on the beam section.

The load curve of figure 2 is well fitted with the parameter values: $E_{0}=4.70 \mathrm{GPa}, a=0.845$ and $b=0.0013$. The $E_{0}$ value is consistent with uniaxial test data and has been confirmed by an ultrasonic measurement which led to an Young modulus of $4.69 \pm 0.15 \mathrm{GPa}$. These elastic and damageable laws for aluminium titanate are mechanically admissible, so they can directly be used in a finite-element numerical simulation.

Whereas elastic linear formulae gave fracture strengths of $\pm 5.0 \mathrm{MPa}$ in tension and in compression, the identified constitutive law leads to a strength of $+3.5 \mathrm{MPa}$ for tension and a

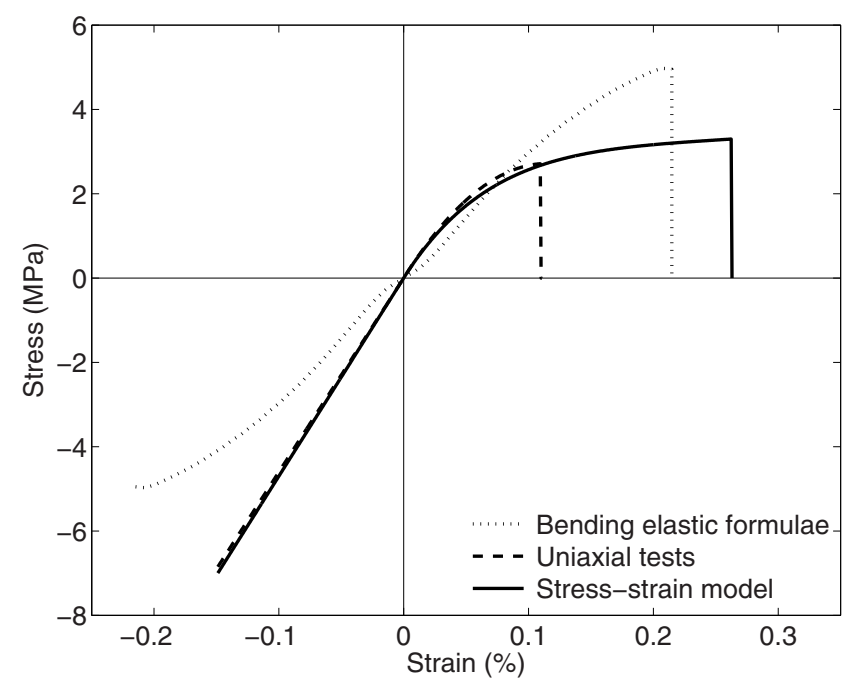

Figure 6. Measured and identified stress-strain curves for aluminium titanate 
stress of $-7.1 \mathrm{MPa}$ for compression at failure (Figure 6). Relative errors of 30-40\% were thus done with the elastic formulae.

\section{Conclusion}

DIC is an efficient optical technique for full field strain measurements, especially when associated with a specific displacement decomposition. It enables one to introduce mechanical hypotheses into a numerical problem. Two specific basis function sets have been developed: a constant curvature element for four-point bending tests (beam-DIC approac) and a homogeneous strain element for uniaxial tests (uni-DIC approach).

The specific tools developed for DIC have been applied to aluminium titanate at room temperature. During a four-point bending test, the non centered neutral axis position indicated a difference between tension and compression. Uniaxial tests revealed that the mechanical behaviour can be considered as elastic in compression and as damageable in tension because of the micro cracks propagation. A stress-strain model and its damage law have been identified and validated by successfully predicting the load curve of the bending test. A difference of 30-40\% was observed for fracture strengths between the identified constitutive laws and the elastic linear formulae.

This methodology brings a new alternative to characterize quasi brittle ceramics instead of using unadapted linear formulae. It enables a direct identification of uniaxial stress-strain models from a classical bending test. Beyond the aluminium titanate, it can be applied to other quasi brittle materials among biomaterials, cements, plasters, concrete, composites, rocks and porous ceramics.

\section{Acknowledgements}

The authors wish to thank Fabiano Rodrigues and his colleagues at the CREE (Saint-Gobain group) for providing the samples and financially supporting this research project.

\section{References}

1. Kachanov, M. Mechanics of Materials 1(1), 29-41 (1982).

2. Swain, M. Engineering fracture mechanics 40(4-5), 871-877 (1991).

3. Thomas, H. and Stevens, R. British ceramic. Transactions and journal 88(4), 144-151 (1989).

4. Laws, V. Journal of Materials Science 16(5), 1299-1304 (1981).

5. Sutton, M., Wolters, W., Peters, W., Ranson, W., and McNeill, S. Image Vision Comput 1(3), 133-139 (1983).

6. Hild, F. and Roux, S. Strain 42, 69-80 (2006).

7. Hild, F., Roux, S., Gras, R., Guerrero, N., Marante, M., and Florez-Lopez, J. Optics and Lasers in Engineering 47(3-4), 495-503 (2009).

8. Besnard, G., Hild, F., and Roux, S. Experimental Mechanics 46(6), 789-803 (2006).

9. Roux, S. and Hild, F. International Journal of Fracture 140(1), 141-157 (2006).

10. Chen, C. and Awaji, H. Journal of the European Ceramic Society 27(1), 13-18 (2007).

11. Bazant, Z. and Li, Z. Journal of Structural Engineering 121(4), 739-746 (1995).

12. Swain, M. Journal of the American Ceramic Society 73(3), 621-628 (1990). 